\title{
CHALLENGES AND OPPORTUNITIES: TRANSITION TO ONLINE STREAMING PERFORMANCES IN THE SOUTH AFRICAN OPERA INDUSTRY
}

\author{
Yende SJ* \\ South African College of Music, University of Cape Town, South Africa
}

\begin{abstract}
The COVID-19 pandemic has caused a profound sense of uncertainty and insecurity in the world of opera. The COVID-19 pandemic has emerged as a phenomenon that is brutally affecting the opera industry. This effect was most prevalent and predominant in developing countries. Within a short period, the COVID-19 pandemic triggered unprecedented turmoil in the world of the arts. In European countries, many well-established opera companies opted for online streaming. The seismic disruption brought by the protocols imposed by the COVID-19 pandemic confirms the importance of changing from the traditional method of performance. Hence, this paper explores the challenges and opportunities of the transition to online streaming performances in the South African opera industry. A qualitative research method was employed using reviews of scholarly writings and informal interviews with opera singers and managers of opera companies. A thematic analysis was adopted to analyse the findings. The findings were presented in themes, namely 1 . Challenges and opportunities of the transition to online streaming performance; 2. Effect of the national lockdown in South African opera industry; 3. Vulnerability of opera singers in the South African opera industry. The findings demonstrate that the South African opera industry was hit hard by the pandemic. The severity of the impact is partly attributable to the lockdown the South African government imposed to curb the spread of coronavirus. This paper concludes by stating that online streaming performances could be a longstanding solution for the opera industry.
\end{abstract}

Keywords: COVID-19 pandemic, challenges, opera industry, opportunities, online streaming, lockdown, vulnerability

\section{Introduction}

The South African opera industry is still witnessing unprecedented challenges and changes since the onset of the coronavirus (COVID-19) pandemic. The COVID-19 pandemic began in China and grew to be a worldwide crisis that affected all sectors. In South Africa, the COVID-19 pandemic gained momentum early on 5 March 2020, which led to the announcement of the national lockdown that commenced on 27 March 2020 (Mahaye, 2020). The COVID-19 pandemic brought seismic changes to opera worldwide, forcing the industry to suspend traditional activities such as live performances (Banks, 2020; Tsioulakis and FitzGibbon, 2020). Across the globe, the opera industry was seriously disturbed by the inevitable changes brought by the COVID-19 pandemic (Sataloff, Naunheim and Carroll, 2020). Hence, the European opera industry opted for online performances to ensure that the opera industry was not lost. 
Since the beginning of the worldwide lockdown, many opera artists have increasingly found it challenging to continue with traditional performances due to the implementation of social distancing (Banks, 2020; Tsioulakis and FitzGibbon, 2020). The European opera industry opted for online performances to ensure that the opera industry was not lost. Published scholarly writings give glimpses about the impact of the COVID-19 pandemic on the performing arts industry worldwide (Banks, 2020; Mahaye, 2020; Sahu, 2020; Tsioulakis and FitzGibbon, 2020). Limited attention was given to these investigations into the COVID-19 pandemic in the South African opera industry and its potential implications in the future.

In the context of South Africa, the implementation of social distancing measures resulted in the closure of small and large opera theatres. Private and public venues were affected by the national lockdown. Due to the national lockdown, various opera companies in South Africa suspended their annual performances and concerts. The suspension of the performances and concerts led to the termination of contracts for opera artists (Primov-Fever, Roziner and Amir, 2020).

This paper is motivated by the appraisal made by Tsioulakis and FitzGibbon (2020), who establish that the transition to online streaming performances could improve the opera industry worldwide. Recently, online live-streaming performances became a global trend for the opera industry (Sataloff, Naunheim and Carroll, 2020). The existing literary writings reveal that the appraisal of the online live-streaming performances for the opera industry became a significant point of interest for researchers and scholars (Sataloff, Naunheim and Carroll, 2020; Tsioulakis and FitzGibbon, 2020). In European countries, opera houses that transitioned to online streaming performances continued with operations (Tsioulakis, Bastani, Linardou, \& Sharafi, 2021).

South African opera companies opted for transition to online live-streaming performances to continue with operations during this unprecedented era. There is a necessity to explore the challenges and opportunities in the South African opera industry further regarding the transition to online streaming performances.

\section{The purpose of this study}

- To explore the challenges and opportunities of the transition to online streaming performances.

- To identify and describe the effect of the national lockdown in the South African opera industry.

- To discuss the economic vulnerability of opera singers in the South African opera industry.

\section{Literature review}

\section{The impact of the COVID-19 pandemic in the performing arts}

Many studies found that since the outbreak of the COVID-19 pandemic, countries worldwide opted for stringent restrictions on traditional social activities to curb the speed of transmission of the coronavirus (Tsioulakis and FitzGibbon, 2020). Banks (2020) highlights that the impact of the COVID-19 pandemic caused a seismic disruption in all sectors, reducing the productivity of the usual 
business day. In contrast, Mahaye (2020) believes that the stringent restrictions imposed by the government in several countries were necessary to restrain the rapid spread of coronavirus.

Certainly, just like many other sectors in South Africa, the COVID-19 pandemic seriously impacted the performing arts (South African Cultural Observatory, 2020). The COVID-19 pandemic caused theatres, cinemas, and other entertainment venues to shut down to adhere to the social distancing measures imposed (Primov-Fever, Roziner and Amir, 2020; Sataloff, Naunheim and Carroll, 2020). The swift change from the normal way of living to the new way of living brought profound obstacles and challenges in the performing arts industry. Many performing artists lost their jobs (Tsioulakis and FitzGibbon, 2020). As stringent restrictions continue in many countries, performing artists are continuing to lose their jobs.

Although the performing arts industry is one of the main industries contributing to the economy of South Africa, the COVID-19 pandemic has adversely affected the industry and led to the severe stagnancy of the country's economy. Unfortunately, medical experts have attested that no one knows when the COVID-19 pandemic will be alleviated and the experts continue to advise for the suspension of the public gatherings (Primov-Fever, Roziner and Amir, 2020; Tsioulakis and FitzGibbon, 2020). Since the performing arts industry relies on the public gathering for generating revenues, the continuation of the suspension of the public gatherings means that performing artists cannot generate any income (Tsioulakis and FitzGibbon, 2020).

\section{The efficiency of the online platforms for the opera industry}

Due to the lockdowns, the global performing arts industry increasingly battles to continue performing live (Dance and Musical Theatre, Music, and Opera) (Sataloff, Naunheim and Carroll, 2020; Tsioulakis and FitzGibbon, 2020). The COVID-19 pandemic brought about visible challenges in the performing arts industry.

Many in the industry have transitioned to online performances in European countries to continue making a living (Primov-Fever, Roziner and Amir, 2020; Tsioulakis and FitzGibbon, 2020). Hence, Tsioulakis and FitzGibbon (2020) state that digital performances were deemed effective for performers and the performing arts industry during this trying time.

The COVID-19 pandemic presented opportunities for the performing arts industry to continue with short online gigs using various social media platforms and sending professional performance videos, such as voice-over singing and video performances (Sataloff, Naunheim and Carroll, 2020; Tsioulakis and FitzGibbon, 2020). The COVID-19 pandemic reinforced the importance of the transition to digital performances using online platforms in a changing South African opera performance terrain.

\section{Social and global-distancing factors in the performing arts industry}

Several studies recognise that performing artists worldwide are battling to make a living due to the enforcement of strict precautions to curb the spread of coronavirus. The precautions are a significant problem since performing artists survive by performing live. This problem is heightened by the odd circumstances of global distancing and the swift pace of change in the wider global economic arena (Banks, 2020; Primov-Fever, Roziner and Amir, 2020; Tsioulakis and FitzGibbon, 2020). 


\section{Conceptual framework}

This paper explores the challenges and opportunities of the transition to online streaming performances in the South African opera industry. Therefore, this paper uses the online-performance concept as a lens. The online-performance concept was devised by Elena Khokhlova in her published 2018 doctoral thesis, Opera houses online: Marketing sophisticated art in the age of big data and social media. The thesis significantly impacted the opera industry during this pandemic era Khokhlova proposes and recommends online-live streaming and provides a comprehensive understanding of the importance of live streaming in the opera industry. Khokhlova (2018) proposes that online live-streaming performances are an effective strategy to attract new audiences while reducing the challenges faced by the opera industry. Khokhlova's concept was echoed by Tsioulakis and FitzGibbon (2020), who claim that the transition to online live-streaming performances for the opera industry was necessary and valuable for opera houses. The concept of online live-streaming performances presents the opera industry with a lifelong ultimate strategy for survival in the future. Thus, generally, most European opera houses have successfully transitioned to online live-streaming performances.

In the context of South Africa, there is a necessity for the opera industry to transition to online streaming performances to improve and help the industry to continue with its operation. In this paper, the transition to online streaming performances in the opera industry is perceived as a relevant concept to address the challenges experienced by the industry. Transition to online live-streaming performances is perceived as a fundamental tool to bring opportunities to the South African opera industry to increase its revenues. Thus, online live-streaming performances in the South African opera industry are suggested by this paper to be the cornerstone for the revitalisation of the industry. Therefore, the strength of this concept of a transition to online live-streaming performances was explaining how opera companies should operate. In short, online live-streaming is a concept that seeks to improve the opera industry during the pandemic and in the future.

\section{Methodology}

The descriptive phenomenology design was used for this study to address the research objectives successfully. This design was selected because the focus of this study was on describing the transition to online-streaming performances to establish a practical solution to the problem of no live performances for the opera industry.

Also, the justification for selecting a descriptive phenomenology design was that it helps obtain an indepth understanding of the experiences of opera singers and opera company managers as they explain their experiences with the transition to online-streaming performances. The design was considered suitable as this paper also sought to broaden the understanding of the challenges and opportunities of the transition to online streaming performances in the South African opera industry.

The sample consisted of ten opera singers and two opera company managers from two provinces in South Africa, namely Gauteng and the Western Cape. The participants were informally interviewed via phone. All the participants experienced challenges with the transition to online streaming performances. They embraced the transition to online streaming performances. These participants were selected using purposeful and snowball sampling techniques. These techniques were appropriate 
due to the participants being in various places because of the COVID-19 pandemic. The only means of contacting the participants was through other research participants' assistance and the researcher's knowledge. Upon reaching a participant via various channels such as Microsoft Teams, Skype, Zoom, Google Duo, and phone, the participants were briefly informed about the nature and purpose of this study. Their consent was sought before the interview phase.

Data analysis started after the transcription of the interviews. The researcher employed Colaizzi's phenomenological data analysis using the seven-step method (Wirihana, Welch, Williamson, Christensen, Bakon and Craft, 2018). Edward and Welch (2011) define Colaizzi's phenomenological data analysis as a rigorous and vigorous method of analysis that assures the dependability, trustworthiness, and reliability of the study's findings. In this paper, the researcher used sequential letters of the alphabet instead of the participants' initials to ensure anonymity, privacy, and confidentiality. The study's findings were interpreted and presented in themes and supported by quotations from the participants. Furthermore, a review of scholarly writings such as articles, theses, and other relevant documents was necessary to provide trustworthy findings (Edward and Welch, 2011).

\section{Ethical clearance}

In this paper, a letter of consent was received from the research ethics committee at the University of Cape Town. All the necessary submissions for ethical clearance were submitted to the research committee. The reference number for the clearance is HDC REC 04/2021.

\section{Results}

Perhaps it is prudent to foreground the unprecedented impact of the COVID-19 pandemic in the opera industry worldwide that ushered performing artists into a new territory of human history. The preliminary findings of this paper highlight that termination of performances in the opera industry led to the unemployment of many performing artists. This unemployment was because many performing artists are freelancers and contractors.

This section highlights the findings presented in this paper as themes that emerged from the 12 selected participants who were opera singers and opera company managers. Scholarly writings complemented the findings.

The findings are divided into two main sections, namely:

- Opera singers' perspective

- Managers of opera companies' perspective.

The sub-aims were developed according to the literature gathered in this study:

- Challenges and opportunities of the transition to online streaming performance

- Effect of the national lockdown in South African opera industry

- Vulnerability of opera singers in the South African opera industry. 


\section{Opera singers' perspective}

What are the challenges you are experiencing with the online streaming performances?

Participants described that moving to online streaming performances came unexpectedly, and as a result the transition to online streaming performances was not easy. The transition requires much time and financial investment to produce quality performances. Participants explained that online streaming performances are complex to embrace, as most of their audiences are located in various places.

\section{Participant A stated:}

I think moving to online streaming performances is a difficult concept for us as opera singers as there is a lot of challenges with it. Some of these challenges include lack of access to rehearsal facilities.

\section{Participant B highlighted that:}

Online streaming is really challenging as it seriously reduces employment for opera singers because fewer singers are required in online streaming as compared to live performances. It is hard for us to survive in the era of online streaming.

Another participant highlighted that:

Opera singers who are employed on a full-time basis are not affected by the transition from live performances to online performances, but they have continued to benefit as some opera companies continue with online performances. On the other hand, we who are freelancing opera artists are facing challenges in moving to online platforms, due to various reasons and hence we are facing financial difficulties.

The freelancer opera-singer participants highlighted that they experience difficulties with the online streaming performances. Their difficulties are echoed by Tsioulakis, FitzGibbon (2020) and Yende (2021), who agree that most opera singers in South Africa battle to implement online streaming performances. The difficulties are attributed to opera companies and orchestras that reduce the number of opera artists and ensembles required to provide performances in intimate environments with smaller crowds (Yende, 2021).

\section{Managers of opera companies' perspective}

What are the challenges you are facing with online streaming performances?

Company-manager participants state that like many other sectors, the opera industry battles with making the transition to online streaming performances. Participants have pointed out that opera companies battle due to various issues such as financial resources to conduct online streaming performances. 
One of the opera company managers pointed out that:

I believe that opera companies in South Africa are battling to adapt to online streaming performances due to the high cost of managing online streaming performances.

\section{Another opera company manager stated that:}

Although opera companies in Europe are able to adapt to online streaming performances, the case is different in South African opera companies as we experience various challenges with online streaming performances. We need high-quality Internet, data for connection, and other facilities.

Based on the above responses, opera company managers are aware that online streaming performances are expensive and demand proper facilities. This requirement for expensive facilities was also echoed by Yende (2021), who points out that the opera industry in South Africa battles to move to online streaming performances due to the lack of proper facilities to conduct online streaming performances.

\section{Data acquired according to the developed aims using existing literature}

\section{Challenges and opportunities of the transition to online streaming performance}

The challenges brought by the COVID-19 are evident as many employees have lost their jobs in various sectors, especially vulnerable sectors such as sport, hospitality, and performing arts (Tsioulakis and FitzGibbon, 2020). Tsioulakis and FitzGibbon (2020) highlight that:

Cultural work and the focus of our contribution here - performing arts (music and theatre) - is affected more than many other occupations. This is because performing artists work in extremely precarious conditions, their careers and mental health have been made additionally vulnerable by prolonged austerity, and proposed solutions thus far are inadequate and based on misunderstandings. Both immediate action and a long-term approach are needed to ensure a critical workforce is not abandoned.

Performing artists are experiencing a disturbance concerning their employment due to the imposed national lockdown measures to curb the spread of coronavirus. The COVID-19 pandemic negatively affects the lives of performing artists, especially in South Africa (South African Cultural Observatory, 2020). In South Africa, the COVID-19 pandemic has significantly caused a dwindling economy. The effect of the lockdowns on the economy impacts the arts and leaves many artists unemployed.

Despite the gloomy picture portrayed by various scholars concerning the future, the opera industry can be prevented from recession by determining longer-term digital performances (Tsioulakis and FitzGibbon, 2020). Incontrovertibly, the COVID-19 pandemic calls for a shift from traditional performances to digital performances in the opera industry worldwide. Hence, the transition to online live-streaming performances in the South African opera industry could contribute to a sustainable income for the industry.

The advantage of online live-streaming performances is that performing artists can reach their fans conveniently (South African Cultural Observatory, 2020). The performing artists do not require face- 
to-face (live) performances, which are prevented and controlled by lockdown guidelines to curb the spread of coronavirus (Tsioulakis and FitzGibbon, 2020). Therefore, aligning performing artists with digital performances is vital. By doing this, the opera industry can advance the industry for future generations. The transition to the new way of performing could assist the performing artists in putting food on the table. The adaptation to innovative performance platforms brings opportunities for performing artists to have financial security during this cumbersome COVID-19 pandemic.

Online platforms are game-changers regarding the present obstacles experienced by the opera industry, especially in developing countries. However, obstacles such as restrictions on data and Internet connectivity are hot-button issues that still affect the industry (Sataloff, Naunheim and Carroll, 2020; Tsioulakis and FitzGibbon, 2020). Social media platforms such as Skype, Zoom, Facebook, and Instagram are regarded as a lifeline for marketing and an innovative way of online live streaming for performers. However, these platforms require performing artists to be committed to understanding the online operation of the performing arts and the media (Primov-Fever, Roziner and Amir, 2020).

\section{Effect of the national lockdown on the South African opera industry}

In an attempt to limit the spread of coronavirus, policymakers and the government imposed a national lockdown. The implementation of lockdown negatively affected the economy and the opera industry, particularly in South Africa. The lockdown meant that all the theatre-based sectors, including museums, performing arts, live music, festivals, and cinema had to suspend their operations to adhere to the government restrictions (South African Cultural Observatory, 2020). The national lockdown resulted in reduced incomes in the opera industry, and some opera singers permanently lost their jobs. Many opera singers have to find new forms of income during the lockdown. At the same time, various independent opera companies in South Africa struggle to stay afloat.

\section{Vulnerability of opera singers in the South African opera industry}

In this study, it cannot be overemphasised that the COVID-19 pandemic changed the traditional operations of the opera industry, leaving the performing artists economically vulnerable. As the COVID-19 pandemic spread, theatres and venues canceled events. These cancellations meant that musicians and theatre artists immediately lost their jobs (Primov-Fever, Roziner and Amir, 2020). The termination of opera productions in the industry due to the pandemic significantly affected the lives of artists worldwide. The COVID-19 pandemic brought severe economic vulnerability to performing artists worldwide (Primov-Fever, Roziner and Amir, 2020).

\section{Tsioulakis and FitzGibbon (2020) further state:}

Additionally, for many performing artists who self-produce through small independent unstaffed companies, there are loan liabilities pending taken against predicted bookings, and future contracts to pay for necessary advance costs like PR, equipment hires, and so on. There are too the lost opportunities that for many will be career-changing: a sell-out show, a leading role, a breakthrough album, a concert tour that may never be recovered. 
Since the announcement of the South African national lockdown, the performing arts and opera theatres suspended operations in response to outbreaks of coronavirus. The temporary closure of the opera industry due to the COVID-19 pandemic led to many performing artists becoming an economically vulnerable group, as many are freelance artists. Tsioulakis and FitzGibbon (2020) recently reported that the rapid spread of coronavirus poses a severe threat to the lives of performing artists worldwide.

\section{Discussion}

The current study sought to understand the challenges and opportunities for the transition to online streaming performances in the South African opera industry. This paper argued that although the transition to online live-streaming performances presents a significant opportunity to transform the South African opera industry, freelancers experience resource challenges regarding online streaming performances. The paper also highlighted that the national lockdown negatively impacts the economy of the opera industry in South Africa. This paper noted that freelancing opera artists are economically vulnerable compared to those who are employed full-time.

The research participants described the transition from live performances to online performances as a daunting challenge for both opera artists and opera companies. Consistent with previous studies by Tsioulakis, FitzGibbon (2020) and Yende's (2021), this paper's findings reveal that the COVID-19 pandemic presents serious challenges to the opera industry worldwide, leaving many opera artists unemployed. Evidently, the transition from live performances to online live-streaming performances is difficult for many opera artists. This study findings show that opera companies have to accommodate freelance opera artists.

The findings of this paper demonstrate that online performances are fundamental for the opera industry to continue with its operations. Hence, this paper affirms a necessity for the opera industry to embrace and adapt to online streaming performances. This finding is in aligns with the concept of online streaming performances that assists the opera industry in reaching a new audience into the opera industry.

Again, the findings of this paper show that online performances brought opportunities for the opera industry to sell tickets and generate some revenue through platforms such as Zoom, Facebook, Instagram, and other web-based resources for live concerts. This finding corroborates with the results of Yende (2021), who recently found that it was common for opera artists and the opera industry to use platforms such as Zoom, Facebook, Instagram, and other web-based resources to continue with their performances. These platforms brought opportunities for freelancing opera artists to use virtual platforms as a source of income. This paper established that although there are challenges with the transition to online performances, there are also opportunities for opera artists and the industry. This finding is congruent with findings of previous studies by Tsioulakis, FitzGibbon (2020), and Yende (2021) that suggest that the opera industry should adapt to online streaming performances and find innovative ways to survive during the pandemic.

The results of this study reveal that the transition to online streaming performances in the South African opera industry will enable both opera companies and performing artists to have a sustainable income. These findings imply that the transition to online streaming performances in the South African opera industry is desirable in the era of COVID-19. 


\section{Recommendations}

Based on these findings and reflecting on this study, the following recommendations are made: Firstly, opera companies and opera artists should adapt to online streaming performances to sustain their operations. This use of online streaming will also assist freelancing opera artists to do online private performances. Secondly, the government and other relevant public funders have to provide sufficient relief grants for opera artists and opera companies to revive the opera industry that is losing significant revenue during the pandemic. Lastly, the researcher recommends that other scholars investigate further attributes of the online streaming performances for a broader spectrum of South African performing artists. Undeniably, that the COVID-19 pandemic severely disturbed economic, social, and political environments worldwide. These environments are constantly confronted, and research would better prepare the opera industry and performing artists for this new era in human history.

\section{Conclusions}

This paper reports on a study that explores the challenges and opportunities of the transition to online streaming performances in the South African opera industry. Specific focus was placed on opera artists and opera companies in South Africa. This study looked broadly at the challenges and opportunities regarding the transition. This study recognised that online streaming performances are a fundamental strategy for the continued operation of the opera industry during the pandemic. This study also points out that online streaming performances are imperative for freelancing opera artists, as it enables the artists to perform on virtual platforms.

This paper's conceptual framework of analysis was largely informed by the central tenets of Khokhlova's (2018) concept of online live-streaming performances. This study examined the importance of online performances during the pandemic era. In so doing, this paper argues and reveals that adapting to online performances is vital to increasing the income of the opera industry. The approach and analysis in this paper were informed by the progressive developed in the opera industry as an essential key for opera companies worldwide.

Increasingly, online streaming performances can transform the South African opera industry and its economy. The COVID-19 pandemic visibly impacted every sphere of the performing arts, including the opera industry. Online streaming performances could significantly transform the performing arts industry. Meanwhile, most people stay in their homes due to the restrictions imposed to curb the spread of coronavirus. This paper concludes by stating that online streaming performances can be a longstanding solution in the opera industry.

\section{Acknowledgments}

The financial assistance of the National Institute for the Humanities and Social Sciences, in collaboration with the South African Humanities Deans Association (SAHUDA) towards this research is hereby acknowledged. Opinions expressed and conclusions arrived at are those of the author and are not necessarily to be attributed to the NIHSS and SAHUDA. 


\section{Declaration of interest statement}

The author declares that there is no conflict of interest.

\section{References}

Banks, M. (2020) 'The work of culture and C-19', European Journal of Cultural Studies. doi: $10.1177 / 1367549420924687$.

Belk, R. W. (2017) 'Qualitative research in advertising', Journal of Advertising, 46(1), pp. 36-47.

Edward, K. L., \& Welch, T. (2011). The extension of Colaizzi's method of phenomenological enquiry. Contemporary nurse, 39(2), 163-171.

Khokhlova, E., 2019. Opera houses online: marketing sophisticated Art in the age of big data and social media. Doctoral degree at Masaryk University in Czech. Available on: https://is.muni.cz/th/j8gj6/THESIS.pdf

Mahaye, N.E. (2020) The Impact of COVID-19 Pandemic on South African Education: Navigating Forward the Pedagogy of Blended Learning The Impact of COVID-19 Pandemic on Education: Navigating Forward the Pedagogy of Blended Learning Ngogi Emmanuel Mahaye Director: Office of , (April).

Primov-Fever, A., Roziner, I. and Amir, O. (2020). 'Songbirds Must Sing: How Artistic Voice Users Perceive Their Voice in Times of COVID-19', Journal of Voice. Elsevier Inc. doi: 10.1016/j.jvoice.2020.07.030.

Sataloff, R. T., Naunheim, M. R. and Carroll, T. L. (2020). 'Singing and the Pandemic: Return to Performance?', Journal of Voice. Elsevier Inc., pp. 1-2. doi: 10.1016/j.jvoice.2020.07.031.

South African Cultural Observatory (2020). Impact Analysis Live Music and its Venues and the South African economy during COVID-19. Report Retrieved from https://www.southafricanculturalobservatory.org.za/download/comments/691/10a5ab2db37feedfdeaa b192ead4ac0e/Impact+Analysis+-

+Live+Music+and+its+Venues+and+the+South+African+economy+during+COVID-19

Tsioulakis, I. and FitzGibbon, A. (2020, 09 April). Performing Artists in the age of COVID-19: A moment of urgent action and potential change. Retrieved from http://qpol.qub.ac.uk/performingartists-in-the-age-of-covid-19/

Tsioulakis, I., Bastani, H., Linardou, A., \& Sharafi, R. (2021). Musical careers in constant crises. Critical Studies in Improvisation/Études critiques en improvisation, 14(2-3).

Wirihana, L., Welch, A., Williamson, M., Christensen, M., Bakon, S., \& Craft, J. (2018). Using Colaizzi's method of data analysis to explore the experiences of nurse academics teaching on satellite campuses. Nurse Researcher (2014+), 25(4), 30.

Yende S.J. (2021). A glimpse of response of the South African performing arts industry to the COVID-19 pandemic. A Re Bueng Seminars. Hosted by the Tshwane University of Technology Virtual on the 17 September 2021. 